\title{
Energy relaxation mechanism of hot-electron ensembles in GaAs: Theoretical and experimental study of its temperature dependence
}

\author{
Jelena Sjakste, ${ }^{*}$ Nathalie Vast, and Giuliana Barbarino \\ Ecole Polytechnique, Laboratoire des Solides Irradiés, CEA-DRF-IRAMIS, CNRS UMR 7642, \\ Paris-Saclay University, 91120 Palaiseau, France \\ Matteo Calandra \\ Sorbonne Université, CNRS, Institut des Nanosciences de Paris, UMR7588, F-75252, Paris, France \\ Francesco Mauri \\ Dipartimento di Fisica, Università di Roma La Sapienza, Piazzale Aldo Moro 5, I-00185 Roma, Italy \\ Junichi Kanasaki \\ Institute of Scientific and Industrial Research, Osaka University, 8-1 Mihogaoka, Ibaraki, Osaka 567-0047, Japan \\ Hiroshi Tanimura and Katsumi Tanimura \\ Research Center for Ultra-High-Voltage Electron Microscopy, Osaka University, 7-1 Mihogaoka, Ibaraki, Osaka 567-0047, Japan
}

(Received 24 January 2017; revised manuscript received 26 January 2018; published 6 February 2018)

\begin{abstract}
We have recently demonstrated that the fast momentum relaxation due to electron-phonon scattering of hot electrons excited into the conduction band of GaAs leads to the formation of hot-electron ensembles spread over the Brillouin zone. In the present work, we study the energy relaxation of hot-electron ensembles in GaAs, by means of $a b$ initio calculations and time-, energy-, and momentum-resolved spectroscopy. We theoretically show that when the temperature decreases, the energy relaxation time ascribed to electron-phonon interaction becomes faster than at ambient temperature and prove that this is indeed the case in the experimental results.
\end{abstract}

DOI: 10.1103/PhysRevB.97.064302

\section{INTRODUCTION}

Ultrafast scattering of highly energetic carriers in the conduction band of semiconductors is the key process that ultimately determines the functional limits and properties of micro- and optoelectronics, a strategic research field for the past two decades [1,2]. The development of ultrafast optical spectroscopy over the past 40 years [3-5] has complemented transport measurements by capturing scattering dynamics of the electronic system. Adding to the accumulating knowledge obtained with optical spectroscopy and transport measurements, recent progress both in time- and angle-resolved photoemission spectroscopy (TR-ARPES) [5-7] and in theoretical calculations made from the first principles [8-14] has made it possible to gain insight into electron relaxation dynamics. TRARPES gives direct experimental information on the dynamics of the electronic states resolved in momentum and energy spaces, while theoretical studies using $a b$ initio calculations provide insight into the relative roles of different relaxation mechanisms in detail, thus allowing access to information beyond the one available from experiments [8-14].

At low carrier concentrations, the main mechanism governing the hot-electron relaxation is the interaction of electrons

*jelena.sjakste@polytechnique.edu with lattice vibrations (phonons). A detailed review describing recent progress in the ab initio calculations of the electronphonon coupling can be found in Ref. [15]. The electronphonon coupling in GaAs was actively studied from the $a b$ initio point of view in recent works of Refs. [7,8,13,16,17], as well as in the earlier works of Refs. [18,19]. Nevertheless, so far, $a b$ initio calculations mostly described the electron-phonon relaxation via a self-energy related to electron-phonon interaction $[9,17,18,20-23]$. The imaginary part of the electron-phonon self-energy, i.e., the total probability of electron-phonon scattering for a given electronic state, corresponds to the width of the corresponding absorption peaks due to electron-phonon interaction [24-27], while the real part yields the shifts of the electronic levels [28]. As the inverse of the total probability of electron-phonon scattering yields the relaxation time of the initial momentum of hot electron [7], the imaginary part of the electron-phonon self-energy provides a characteristic for the physical processes governed by momentum relaxation of hot electrons. Indeed, by combining the results obtained by TR-ARPES and numerical calculation from the first principles, it has been recently shown that efficient momentum relaxation due to electron-phonon scattering of hot electrons excited into the $\Gamma$ valley of GaAs leads to the formation of hot-electron ensembles (HEEs) quasiequilibrated over the Brillouin zone (BZ) in a very short time [7]. The probability of the electronphonon scattering grows rapidly with temperature, as the 
phonon occupation number increases, and thus the relaxation times of electrons related to the electron-phonon scattering become faster at higher temperatures [7,29].

In wide contrast to the momentum relaxation rate of hot electrons, for which the total probability of all of the (emission and absorption) electron-phonon scattering processes is the key quantity [30], the energy relaxation rate is determined by the difference between phonon-emission and phonon-absorption rates. Indeed, while phonon emission leads to energy loss by electrons, phonon absorption leads, on the contrary, to energy gain [31,32]. Moreover, the phonon energy plays an important role in the energy relaxation: scattering of electrons by low-frequency phonons results in a quasielastic scattering with a smaller contribution to the energy relaxation than that of high-frequency phonons. As demonstrated in Ref. [7], the energy relaxation rates of hot electrons at excess energy with regard to the conduction band minimum (CBM) ranging from 0.5 to $0.8 \mathrm{eV}$ are lower than the momentum relaxation rates at the same excess energy by more than a factor of 10 , showing remarkable difference between momentum- and energy-relaxation rates of hot electrons.

Although energy loss mechanisms of hot electrons due to electron-phonon interaction are included in calculations made from first principles [33-35], discussions of the different time scales for momentum and energy relaxation based on first principles are scarce [7,36-38]. The calculated momentum and energy relaxation times have hardly been compared to experiments [7].

In this work, we study the mechanisms of the energy relaxation of hot-electron ensembles in GaAs, using state-of-the-art $a b$ initio calculations and time-, energy-, and angle-resolved spectroscopy experiments. Temperature-dependent changes of the energy-relaxation rate are carefully studied. Surprisingly, the energy relaxation rate is found to increase with decreasing temperature, both in experiment and in our theory of energy relaxation of hot-electron ensembles. This is in wide contrast with the momentum relaxation rate, which is found to decrease with decreasing temperature [7].

The paper is organized as follows: First, we present the theoretical background for the energy relaxation due to electron-phonon scattering, as well as technical details for numerical calculations. Next, we give some details on the experimental determination of the slow component of the electron relaxation times in GaAs. Then, we present our experimental and theoretical results of the energy relaxation time of hot-electron ensembles as a function of the excess energy (excitation energy with respect to the CBM) at 293 and $90 \mathrm{~K}$. Finally, we analyze the relative roles of different electron-phonon scattering processes in the energy relaxation of hot electrons.

\section{THEORY}

We consider electrons excited into the conduction band of a semiconductor. If the excess energy is sufficiently high, the momentum relaxation time due to electron-phonon scattering is very short, of the order of a few dozens of femtoseconds, at room as well as at low temperatures, as shown in our previous work [7]. Fast momentum relaxation leads to the formation of hot-electron ensembles: Electrons can be considered as "spread" over the BZ. In the present work, we focus on the subsequent energy relaxation of hot-electron ensembles, which takes place on a longer time scale, a few hundreds of femtoseconds.

Below, we present the theory of the energy relaxation of hot electrons, which underlies our calculations. The same theory was briefly presented in the Supplemental Material of Ref. [7]. However, we find necessary to present it here in more detail, for the sake of clarity and completeness of present work.

For every initial electronic state $|n, \mathbf{k}\rangle$, characterized by the band index $n$ and wave vector $\mathbf{k}$, we calculate the phonon emission and absorption rates $\Gamma^{\mathrm{em}}$ and $\Gamma^{\mathrm{abs}}$, using Fermi's golden rule and taking into account all processes allowed by energy and momentum conservation:

$$
\begin{aligned}
\Gamma_{n \mathbf{k}}^{\mathrm{em}}= & \frac{2 \pi}{\hbar} \sum_{n^{\prime}} \sum_{\lambda} \frac{1}{N_{q}} \sum_{\mathbf{q} \in B Z}\left|\left\langle n^{\prime}, \mathbf{k}+\mathbf{q}\left|\Delta W_{\mathbf{q}}^{\lambda}\right| n, \mathbf{k}\right\rangle\right|^{2} \\
& \times \delta\left(\varepsilon_{n, \mathbf{k}}-\varepsilon_{n^{\prime}, \mathbf{k}+\mathbf{q}}-\hbar \omega_{\mathbf{q}}^{\lambda}\right)\left[n_{\mathbf{q}}^{\lambda}(T)+1\right], \\
\Gamma_{n \mathbf{k}}^{\mathrm{abs}}= & \frac{2 \pi}{\hbar} \sum_{n^{\prime}} \sum_{\lambda} \frac{1}{N_{q}} \sum_{\mathbf{q} \in B Z}\left|\left\langle n^{\prime}, \mathbf{k}+\mathbf{q}\left|\Delta W_{\mathbf{q}}^{\lambda}\right| n, \mathbf{k}\right\rangle\right|^{2} \\
& \times \delta\left(\varepsilon_{n, \mathbf{k}}-\varepsilon_{n^{\prime}, \mathbf{k}+\mathbf{q}}+\hbar \omega_{\mathbf{q}}^{\lambda}\right) n_{\mathbf{q}}^{\lambda}(T) .
\end{aligned}
$$

Here, $\varepsilon_{n, \mathbf{k}}$ and $\varepsilon_{n^{\prime}, \mathbf{k}+\mathbf{q}}$ are the energies of the initial and final electronic states. $\omega_{\mathbf{q}}^{\lambda}$ is the frequency of the phonon mode $\lambda$ with wave vector $\mathbf{q} . \Delta W_{\mathbf{q}}^{\lambda}$ is the change of the crystal potential due to the atomic motion associated with the phonon $|\lambda, \mathbf{q}\rangle$ (see Ref. [39]), $n_{\mathbf{q}}^{\lambda}(T)$ is the temperature-dependent phonon distribution function (we use Bose-Einstein distribution). $N_{q}$ is the number of points of the $\mathbf{q}$ grid in the BZ over which the integral (sum) is performed numerically.

We also calculate the average loss (gain) of energy between an initial state and the final ones due to emitted (absorbed) phonon energies, as the first moment of the transition probability [36]:

$$
\begin{aligned}
\langle\omega\rangle_{n \mathbf{k}}^{\mathrm{emiss}}= & \frac{2 \pi}{\hbar} \sum_{n^{\prime}} \sum_{\lambda} \frac{1}{N_{q}} \sum_{\mathbf{q} \in B Z} \omega_{\mathbf{q}}^{\lambda}\left|\left\langle n^{\prime}, \mathbf{k}+\mathbf{q}\left|\Delta W_{\mathbf{q}}^{\lambda}\right| n, \mathbf{k}\right\rangle\right|^{2} \\
& \times \delta\left(\varepsilon_{n, \mathbf{k}}-\varepsilon_{n^{\prime}, \mathbf{k}+\mathbf{q}}-\hbar \omega_{\mathbf{q}}^{\lambda}\right)\left[n_{\mathbf{q}}^{\lambda}(T)+1\right] / \Gamma_{n \mathbf{k}}^{\mathrm{em}}, \\
\langle\omega\rangle_{n \mathbf{k}}^{\mathrm{abs}}= & \frac{2 \pi}{\hbar} \sum_{n^{\prime}} \sum_{\lambda} \frac{1}{N_{q}} \sum_{\mathbf{q} \in B Z} \omega_{\mathbf{q}}^{\lambda}\left|\left\langle n^{\prime}, \mathbf{k}+\mathbf{q}\left|\Delta W_{\mathbf{q}}^{\lambda}\right| n, \mathbf{k}\right\rangle\right|^{2} \\
& \times \delta\left(\varepsilon_{n, \mathbf{k}}-\varepsilon_{n^{\prime}, \mathbf{k}+\mathbf{q}}+\hbar \omega_{\mathbf{q}}^{\lambda}\right) n_{\mathbf{q}}^{\lambda}(T) / \Gamma_{n \mathbf{k}}^{\mathrm{abs}} .
\end{aligned}
$$

In order to account, in the electronic relaxation process, for changes in both the emission and absorption rates and in the frequencies of the emitted and absorbed phonons, we define the energy loss and gain rates as

$$
\begin{aligned}
& R_{n \mathbf{k}}^{\text {loss }}=\langle\omega\rangle_{n \mathbf{k}}^{\text {emiss }} \Gamma_{n \mathbf{k}}^{\mathrm{emiss}}, \\
& R_{n \mathbf{k}}^{\text {gain }}=\langle\omega\rangle_{n \mathbf{k}}^{\mathrm{abs}} \Gamma_{n \mathbf{k}}^{\mathrm{abs}} .
\end{aligned}
$$

The energy loss and gain rates depend on the energy of the initial electronic state, $\varepsilon=\varepsilon_{n, \mathbf{k}}$ :

$$
\begin{aligned}
& R^{\text {loss }}(\varepsilon)=\langle\omega\rangle^{\text {emiss }}(\varepsilon) \Gamma^{\text {emiss }}(\varepsilon), \\
& R^{\text {gain }}(\varepsilon)=\langle\omega\rangle^{\text {abs }}(\varepsilon) \Gamma^{\text {abs }}(\varepsilon) .
\end{aligned}
$$


It must be noted that Eq. (6) contains an approximation as, for every $\varepsilon$, an integral over $\mathbf{k}$ vectors should be performed to account for all initial electronic states with $\varepsilon=\varepsilon_{n, \mathbf{k}}$. Instead, in this work, for any $\varepsilon$, we have performed calculations for a single initial electronic state with $\mathbf{k}$ in $\Lambda$ direction inside $L$ valley. We have checked that the choice of the $\mathbf{k}$ vector direction does not affect the final result.

Finally, the measured energy relaxation time depends on a typical energy loss interval $\Delta E$. For a given $\Delta E$, the energy relaxation time is given by

$$
\begin{aligned}
\tau_{E} & =\tau(\varepsilon, \Delta E)=\int_{\varepsilon-\Delta E}^{\varepsilon} d \varepsilon^{\prime} \frac{1}{\langle\omega\rangle\left(\varepsilon^{\prime}\right) \Gamma\left(\varepsilon^{\prime}\right)} \\
& =\int_{\varepsilon-\Delta E}^{\varepsilon} d \varepsilon^{\prime} \frac{1}{\langle\omega\rangle^{\mathrm{emiss}}\left(\varepsilon^{\prime}\right) \Gamma^{\mathrm{emiss}}\left(\varepsilon^{\prime}\right)-\langle\omega\rangle^{\mathrm{abs}}\left(\varepsilon^{\prime}\right) \Gamma^{\mathrm{abs}}\left(\varepsilon^{\prime}\right)} \\
& =\int_{\varepsilon-\Delta E}^{\varepsilon} d \varepsilon^{\prime} \frac{1}{R^{\operatorname{loss}}\left(\varepsilon^{\prime}\right)-R^{\text {gain }}\left(\varepsilon^{\prime}\right)} .
\end{aligned}
$$

In this work, we describe GaAs within the density functional theory (DFT) in the local density approximation (LDA), with the same pseudopotentials as in our previous works $[8,18]$. For the electronic density calculation, we used an energy cutoff value of 45 Ry to limit the size of the plane-wave basis set and a Monkhorst-Pack grid of $12 \times 12 \times 12$ points to sample the BZ. The lattice parameter $a=10.49$ a.u. yields the topology of the conduction band, i.e., the energy order of the $\Gamma, L$ and $X$ valleys, in excellent agreement with experimental results (see Supplemental Material of Ref. [7]).

The electron-phonon matrix elements are calculated using the method of Ref. [8], which allows us to interpolate the electron-phonon matrix elements calculated within the density functional perturbation theory (DFPT) [39] in the space of maximally localized Wannier functions, in polar materials. In addition, we have performed model calculations referred to as "Fröhlich coupling only." These calculations were performed using Vogl's model for the electron-polar optical phonon matrix element $[8,40]$.

The Wannier interpolation of the electronic structure was carried out using a $6 \times 6 \times 6 \mathbf{k}$-point grid centered at $\Gamma$, with 10 Wannier functions and 45 DFT Bloch wave functions. The same $6 \times 6 \times 6$ grid centered at $\Gamma$ was used as initial grid to calculate electron-phonon matrix elements within DFPT, which were then Wannier interpolated using the interpolation method of Ref. [8]. The integrals (1)-(4) were calculated numerically on a $100 \times 100 \times 100$ grid with the $\delta$ function replaced by Gaussian function with converged broadening.

It must be noted that the theory presented by Eqs. (1)(8) contains several crude approximations. Among those, the phonon population is considered to be at thermal equilibrium and described with the Bose-Einstein statistical function. This assumption prevents us from describing effects such as the hot-phonon effect $[41,42]$, for which a nonequilibrium phonon population must be accounted for. However, it is shown below that this level of theory is sufficient to explain the experimental results for the hot-electron relaxation.

\section{EXPERIMENTAL METHOD}

Zn-doped $p$-type GaAs wafers (carrier concentration was $\left.1.5 \times 10^{17} \mathrm{~cm}^{-3}\right)$ were cleaved under ultrahigh vacuum conditions $\left(<5 \times 10^{-11}\right.$ Torr $)$. Atomic structures of the (110) surfaces were characterized in situ by a scanning tunneling microscope prior to photoemission measurements. The (110) surfaces displayed well ordered $(1 \times 1)$ structure with linear $\mathrm{Ga}$ and As rows and with a surface-defect concentration smaller than $0.2 \%$. A laser system, consisting of a Ti-sapphire laser oscillator, a regenerative amplifier, and a tunable optical parametric amplifier, generated 40-fs laser pulses centered at photon energies between 1.7 and $2.4 \mathrm{eV}$. Part of the amplified fundamental output at $824 \mathrm{~nm}$ was used to generate the 50 -fs third-harmonic pulses for probing photoemission. Pump and probe pulses, with a preset time delay $(\Delta t)$, were aligned coaxially and focused on the sample surfaces at $45^{\circ}$ to the normal direction.

For photoemission spectroscopy, a hemispherical electron analyzer (PHOIBOS 100), equipped with an angle-resolved lens mode and a two-dimensional image-type CCD detector, served as the electron spectrometer. The entrance slit of the analyzer was placed on the optical plane (detection plane) defined by the incoming and reflected light. Two-dimensional images of photoelectrons were recorded as functions of energy $\varepsilon$ and of emission angle $\Theta$ with respect to the surface normal along the [001] crystallographic direction (normal to [110] surface). The instrumental energy resolution with fs-probe light was $\Delta E=75 \mathrm{meV}$, while angle resolution was limited in the range of $\pm 0.5^{\circ}$.

Figure 1(a) displays the distribution of hot electrons injected into the $\mathrm{CB}$ by $s$-polarized laser pulses with pump-photon energy $h v_{\text {pump }}=2.30 \mathrm{eV}$ at $90 \mathrm{~K}$. The distribution was measured at $\Delta t=33 \mathrm{fs}$. The scale of $\varepsilon$ is referenced to the CBM, which was determined precisely based on the spectral shape analysis of photoemission from completely thermalized electron distribution measured at $\Delta t=20 \mathrm{ps}$. Based on the Fermi level at $90 \mathrm{~K}$ and ionization energy, together with probephoton energy, the CBM energy with respect to the valence band maximum is evaluated to be $1.50 \mathrm{eV}$, which is higher by $0.07 \mathrm{eV}$ than that at $293 \mathrm{~K}$. The solid (broken) curve displays the $\mathrm{CB}$ dispersion along $\Gamma-L(\Gamma-X)$ direction as a function of $\Theta$. One can see in Fig. 1(a) that part of the electronic packets injected into the $\Gamma$ valley starts to be transferred into the $L$ valley $\left(\Theta>16^{\circ}\right)$. Ultrafast transfer of hot electrons into the $L$ valley (momentum relaxation) is accompanied by a progressive loss of energy by the hot electrons (energy relaxation), the latter being visible on the longer time scales. The same electronic distribution as in Fig. 1(a), but at a longer time delay $\Delta t=113$ fs, is shown in Fig. 1(b). One can see that at $\Delta t=113 \mathrm{fs}$, a significant part of electronic packet is already transferred to the $8 L$ valleys (only one is shown in Fig. 1), and also, that the hot electrons have lost energy with respect to the distribution shown in Fig. 1(a).

For lower $h v_{\text {pump }}=2.07 \mathrm{eV}$, Fig. 1(c) displays the distribution of hot electrons injected into the $\mathrm{CB}$ at $90 \mathrm{~K}$. The distribution was measured at $\Delta t=80 \mathrm{fs}$. Excess energies of hot electrons are lower with respect to the ones shown in Fig. 1(a), and thus the transfer to the $L$ valley is slower and the electrons transferred to the $L$ valley are located at lower 


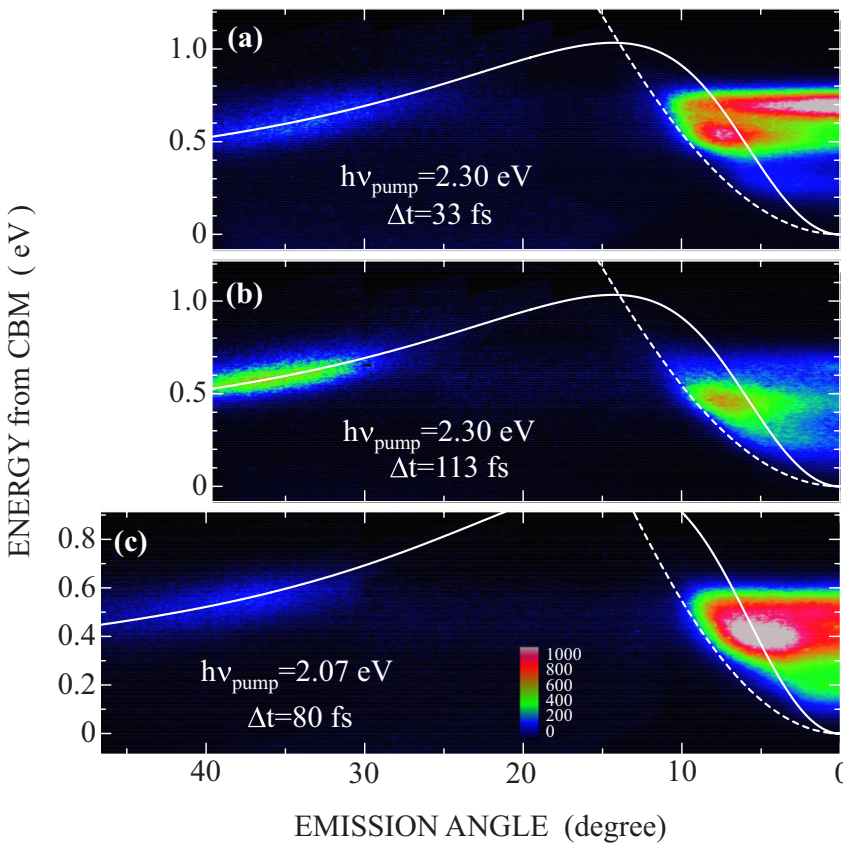

FIG. 1. (a) The photoemission map for hot electrons injected into the conduction band with $s$-polarized $2.30-\mathrm{eV}$ light pulses, at 33 fs after excitation. (b) The photoemission map for hot electrons injected into the conduction band with $s$-polarized $2.30-\mathrm{eV}$ light pulses, at 113 fs after excitation. (c) The photoemission map for hot electrons injected into the conduction band with $s$-polarized $2.07-\mathrm{eV}$ light pulses, at $80 \mathrm{fs}$ after excitation. In panels (a), (b), and (c), measurements were made at $90 \mathrm{~K}$. The solid and broken curves show the dispersion along $\Gamma-L$ and $\Gamma-X$ directions. The color scale indicates the photoemission intensity.

energies than those in Fig. 1(a). Thus, the results in Fig. 1 show that ultrafast momentum scattering, with typical time $\tau_{M}$ of the order of a few tens of fs, is induced at $90 \mathrm{~K}$, similar to the case of $293 \mathrm{~K}$ reported previously [7]. However, both the momentum relaxation time $\tau_{M}$ and the slower time $\tau_{E}$ of the energy relaxation following the momentum relaxation depend strongly on temperature, as shown below.

In Fig. 2, we compare the temporal changes in hot-electron population with $\varepsilon=0.55 \mathrm{eV}$ in the $\Gamma$ valley at 90 and $293 \mathrm{~K}$; Fig. 2(a) shows the changes on a linear scale, while in Fig. 2(b) they are plotted on a semilogarithmic scale. We clearly see a bimodal decay of the population at both temperatures. As shown in the previous paper [7], the fast decay corresponds to the momentum relaxation to form HEEs, while the slow decay represents the energy relaxation of HEEs. However, features of temporal changes are different at lower temperature: The fast decay becomes slower than at ambient temperature, while the slow decay becomes faster. At this excess energy $(\varepsilon=$ $0.55 \mathrm{eV}$ ), the rate of the fast decay is slow enough to enable us to determine the decay times precisely by a simple analysis in the semilogarithmic plot of Fig. 2(b). At $293 \mathrm{~K}, \tau_{M}=31$ fs and $\tau_{E}=300 \mathrm{fs}$, as reported previously [7], while at $90 \mathrm{~K}$ $\tau_{M}=57 \mathrm{fs}$ and $\tau_{E}=220 \mathrm{fs}$.

We measured temperature-dependent changes in both $\tau_{M}$ and $\tau_{E}$ for the range of $\varepsilon$ between 0.3 and $0.8 \mathrm{eV}$. Based on the angle-resolved measurements, the quantities were determined

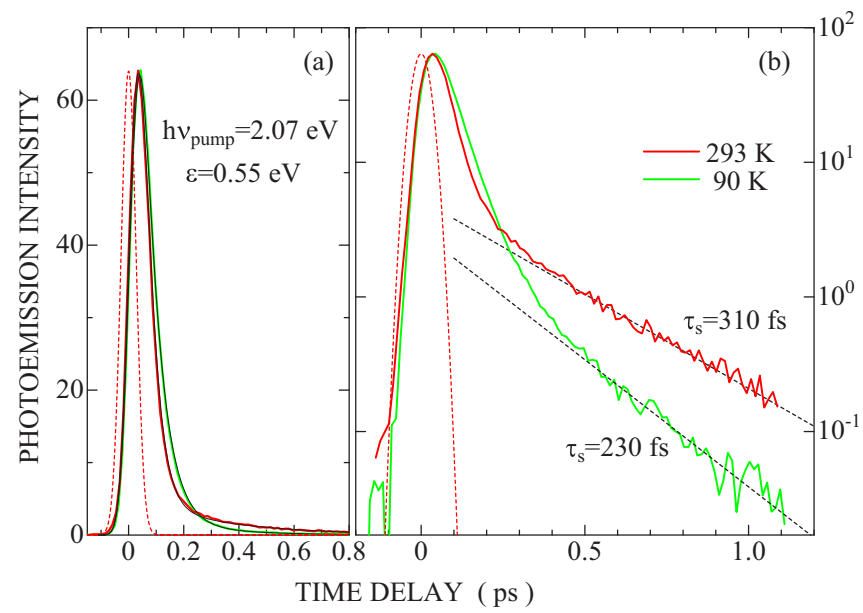

FIG. 2. Temporal changes in hot-electron populations with excess energy of $0.55 \mathrm{eV}$ in the $\Gamma$ valley, measured at 90 and $293 \mathrm{~K}$. The photoemission intensities which represent the populations are plotted on a linear scale in panel (a) and on a semilogarithmic scale in panel (b). The red (dark gray) broken curve shows the cross correlation trace between pump and probe pulses, showing the temporal width of $65 \mathrm{fs}$. The broken lines in panel (b) show the decay of slow components with time constant of 230 and 310 fs at 90 and $293 \mathrm{~K}$. Thin solid curves in panel (a) represent the calculated results of analysis using optical Bloch equation combined with a rate equation (Ref. [5]).

for hot electrons not only in the $\Gamma$ valley, but also in the $L$ valley. The results for $\tau_{M}$ are plotted in Fig. 3. As already mentioned, the momentum relaxation time becomes slower at lower temperatures. The results for $\tau_{E}$ are plotted in Fig. 4. It is clear that at a given temperature, $\tau_{E}$ is the same for hot electrons with a given $\varepsilon$ in both valleys, substantiating our previous conclusion that hot electrons are quasiequilibrated in momentum space via ultrafast momentum relaxation in the whole BZ [7]. It is also clear that the rate of energy relaxation, given by the inverse of $\tau_{E}$, is accelerated at low temperatures. We further discuss this result in the next section.

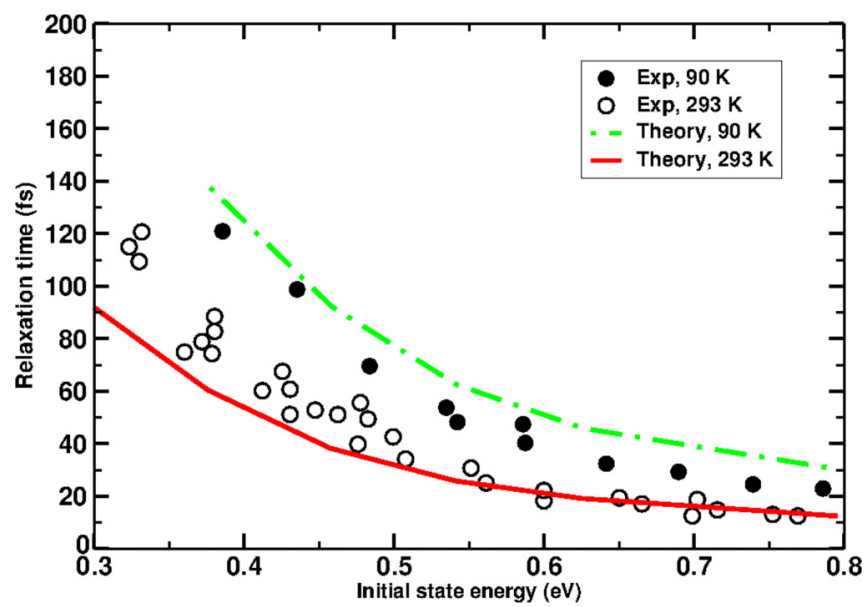

FIG. 3. Momentum relaxation time of hot electrons in GaAs, as a function of the excess energy with respect to the CBM, at $293 \mathrm{~K}$ and 90 K. Empty circles: expt. at 293 K. Full circles: expt. at 90 K. Red solid (green dash-dotted) line: theory at $293 \mathrm{~K}$ (90 K). 


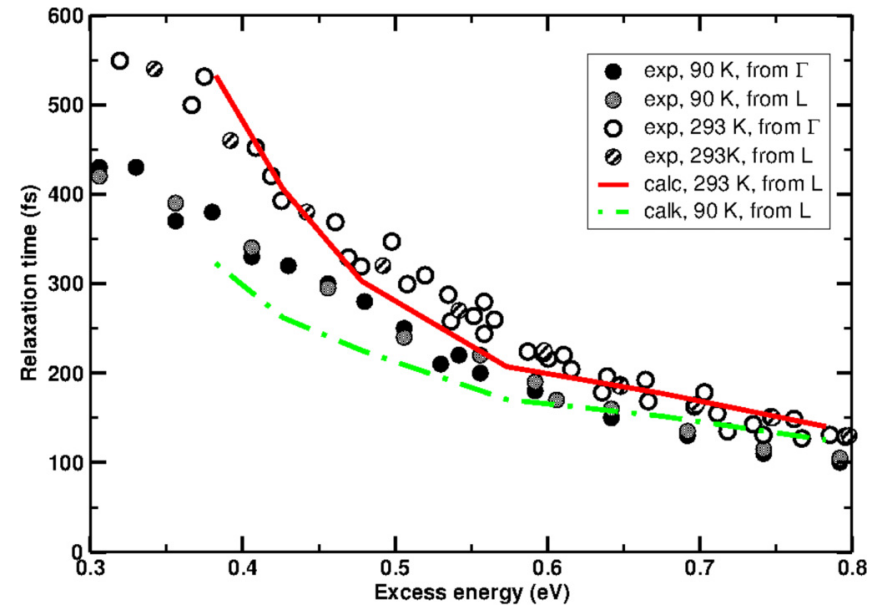

FIG. 4. Energy relaxation time of hot electrons in GaAs, as a function of the excess energy with respect to the CBM, at 293 and 90 K. Empty (stripped) circles: expt. at $293 \mathrm{~K}$, electrons from the $\Gamma$ $(L)$ valley. Full (gray) circles: expt. at $90 \mathrm{~K}$, electrons from the $\Gamma(L)$ valley. Red solid (green dash-dotted) line: theory at $293 \mathrm{~K}(90 \mathrm{~K})$, initial state in the $L$ valley, computed with Eq. (8).

\section{RESULTS AND DISCUSSION}

\section{A. Theory vs experiment}

Although we have discussed the momentum relaxation in Ref. [7], for the sake of completeness in Fig. 3, we show our theoretical and experimental results for the momentum relaxation time of hot electrons in GaAs, at 293 and $90 \mathrm{~K}$, as a function of the excess energy with respect to the bottom of the conduction band. Both theory and experiment agree that the momentum relaxation rate becomes slower at lower temperatures, in agreement with what is expected from the Bose-Einstein statistics.

In Fig. 4, we show our theoretical and experimental results for the energy relaxation time of hot electrons in GaAs, at 293 and $90 \mathrm{~K}$, as a function of the excess energy with respect to the bottom of the conduction band. The experimental energy resolution is $75 \mathrm{meV}$. Thus, we have chosen $\Delta E=75 \mathrm{meV}$ in Eq. (8) to obtain the computed results presented in Fig. 4. For the theoretical curves (solid and dash-dotted lines), the initial electronic wave vector $\mathbf{k}$ was chosen to belong to the $L$ valley. Experimental results are shown for electrons either in the $L$ or $\Gamma$ valleys (respectively, stripped and empty circles).

As one can see in Fig. 4, the agreement between theory and experiment is remarkably good, both for the 293and $90-\mathrm{K}$ curves, supporting the conclusion that the energy relaxation is governed by the electron-phonon interaction and showing that the theoretical framework described by Eqs. (1) to (8) is appropriate to describe the energy relaxation dynamics.

The energy relaxation time strongly varies with the excess energy, from around 700 fs for an excess energy close to the bottom of the $L$ valley, to 100 fs for the excess energy of $0.8 \mathrm{eV}$. The theoretical and experimental dependences of $\tau$ on the excess energy are the same for 293 and $90 \mathrm{~K}$.

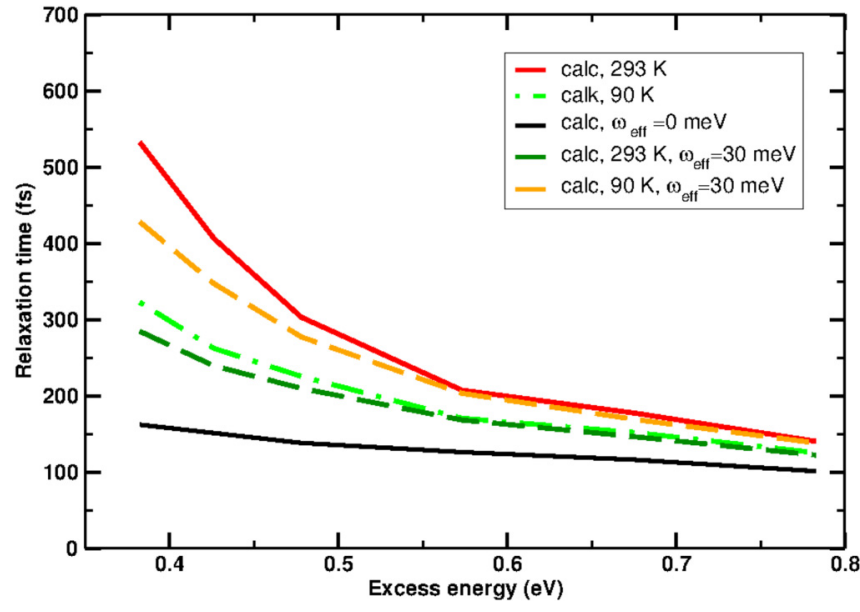

FIG. 5. Energy relaxation time of hot electrons in GaAs, as a function of the excess energy with respect to the CBM, at 293 and $90 \mathrm{~K}$. Red solid (green dash-dotted) line: theory at $293 \mathrm{~K}(90 \mathrm{~K})$, initial state in the $L$ valley, computed with Eq. (8), same as in Fig. 4. Orange and dark green dashed lines: theory at 293 and $90 \mathrm{~K}$, computed with Eq. (8), but with $\omega_{\mathbf{q}}^{\lambda}=\omega_{\text {eff }}=30 \mathrm{meV}$ inside $\delta$ functions [see Eq. (9) and text]. Black line: same, but with $\omega_{\mathbf{q}}^{\lambda}=\omega_{\text {eff }}=0 \mathrm{meV}$.

\section{B. Temperature dependence}

Interestingly, the relaxation times become faster at lower temperatures, both in experiment and in theory. This is due to the density of final electronic states available for emission and absorption. Let us consider the difference between $R^{\text {loss }}$ and $R^{\text {gain }}$, which determines the energy relaxation rate for a given initial state [see Eqs. (1)-(6)]:

$$
\begin{aligned}
R_{n \mathbf{k}}^{\text {loss }}-R_{n \mathbf{k}}^{\text {gain }}= & \frac{2 \pi}{\hbar} \sum_{n^{\prime}} \sum_{\lambda} \frac{1}{N_{q}} \sum_{\mathbf{q} \in B Z} \omega_{\mathbf{q}}^{\lambda}\left|\left\langle n^{\prime}, \mathbf{k}+\mathbf{q}\left|\Delta W_{\mathbf{q}}^{\lambda}\right| n, \mathbf{k}\right\rangle\right|^{2} \\
& \times\left\{\left[\delta\left(\varepsilon_{n, \mathbf{k}}-\varepsilon_{n^{\prime}, \mathbf{k}+\mathbf{q}}-\hbar \omega_{\mathbf{q}}^{\lambda}\right)-\delta\left(\varepsilon_{n, \mathbf{k}}-\varepsilon_{n^{\prime}, \mathbf{k}+\mathbf{q}}\right.\right.\right. \\
& \left.\left.\left.+\hbar \omega_{\mathbf{q}}^{\lambda}\right)\right] n_{\mathbf{q}}^{\lambda}(T)+\delta\left(\varepsilon_{n, \mathbf{k}}-\varepsilon_{n^{\prime}, \mathbf{k}+\mathbf{q}}-\hbar \omega_{\mathbf{q}}^{\lambda}\right)\right\} .
\end{aligned}
$$

Note that if the phonon energy $\hbar \omega_{\mathbf{q}}^{\lambda}$ is neglected in the two $\delta$ functions which correspond to emission and absorption energy conservation ("horizontal" transition approximation), the Bose-Einstein factor $n_{\mathbf{q}}^{\lambda}$ will be canceled out of the expression (9), and no temperature dependence will be found.

Indeed, this can be seen in Fig. 5, where we have shown the results for our calculated energy relaxation times, compared with the calculation in which the phonon energy $\hbar \omega_{\mathbf{q}}^{\lambda}$ was neglected in all the $\delta$ functions of Eq. (9) (black line, labeled $\left.\omega_{\text {eff }}=0\right)$, as well as the calculation in which the phonon energy was taken to be constant and equal to $30 \mathrm{meV}$ (labeled $\omega_{\text {eff }}=$ $30 \mathrm{meV}$ ). As one can see in Fig. 5, the temperature dependence of the calculated energy relaxation times is yielded by the most energetic phonons of typically $30 \mathrm{meV}$, and disappears with vanishing phonon energy.

The temperature dependence which we find is explained by the fact that the density of final electronic states for absorption $\delta\left(\varepsilon_{n, \mathbf{k}}-\varepsilon_{n^{\prime}, \mathbf{k}+\mathbf{q}}+\hbar \omega_{\mathbf{q}}^{\lambda}\right)$ is slightly higher in GaAs at the excess energies under consideration than the one for emission 


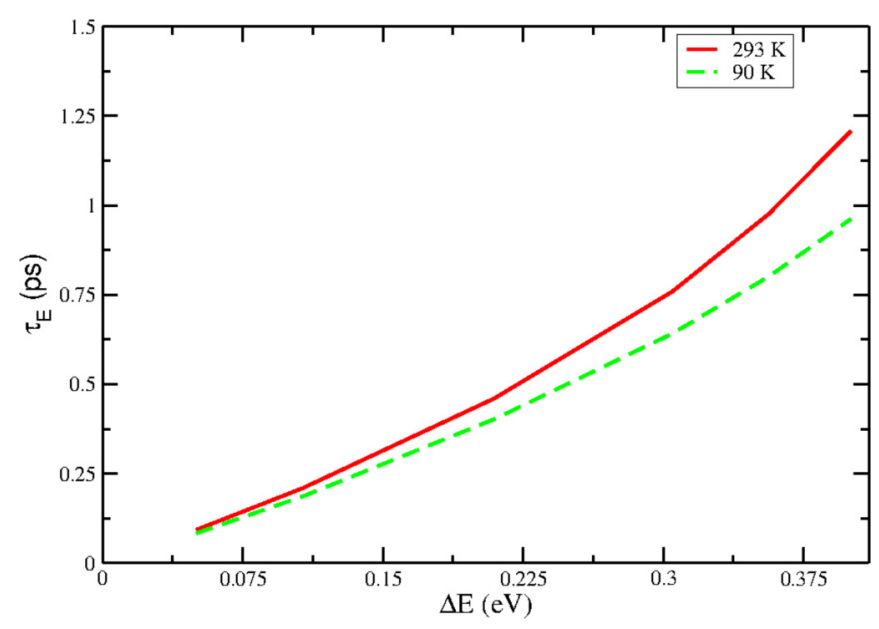

FIG. 6. Calculated energy relaxation time of hot electrons in $\mathrm{GaAs}$, for $\varepsilon=0.78 \mathrm{eV}$ with respect to $\mathrm{CBM}$, as a function of the energy loss interval $\Delta E$. Red curve: $293 \mathrm{~K}$. Green dashed curve: $90 \mathrm{~K}$. Calculations done for an initial electronic state in the $L$ valley.

$\delta\left(\varepsilon_{n, \mathbf{k}}-\varepsilon_{n^{\prime}, \mathbf{k}+\mathbf{q}}-\hbar \omega_{\mathbf{q}}^{\lambda}\right)$, and thus the first (increasing with temperature) term in square brackets in Eq. (9) is negative.

The difference between the density of final electronic states available for emission and absorption depends on the topology of the conduction band and on the phonon energy. The density of final electronic states grows with the excess energy (approximately as the square root of the excess energy in the effective mass model [30]). The phonon energies are determined by the phonon spectrum. As can be seen from Eq. (9), higher phonon energies will yield stronger temperature dependence. In our case, as shown in Fig. 5, the theoretical temperature dependence is almost the same if, instead of the full phonon spectrum, a constant effective frequency of $30 \mathrm{meV}$ is taken into account in the calculated density of final electronic states.

The difference between the density of final electronic states available for emission and absorption is very small, however, and is expected to affect the overall energy relaxation rate when the second (temperature-independent) term in square brackets of Eq. (9) is also small, i.e., only at moderate excess energies. Indeed, in Figs. 4 and 5 one can see that the temperature dependence yields a decrease of the energy relaxation time by as much as $40 \%$ at low excess energies, both in theory and experiment, when the temperature changes from 293 to $90 \mathrm{~K}$. At high excess energies, the experimental energy relaxation time is found to decrease by $15-20 \%$ when the temperature changes from 293 to $90 \mathrm{~K}$, while the theory predicts a $10 \%$ decrease. It must be noted that the excitation energies considered in this work correspond to the range where the density of states in the conduction band still grows rapidly with the electronic energy. The temperature dependence of the energy relaxation rate may be very different or completely absent at energies where the electronic density of states has a plateau at high excess energies.

\section{Relaxation time dependence on the energy loss interval}

In Fig. 4, discussed in previous section, we have shown the energy relaxation time as a function of the electron excess energy, for a given energy relaxation interval $\Delta E$ chosen to be equal to the experimental resolution. In Fig. 6, we show the calculated $\tau_{E}$ of Eq. (8) for a fixed excess energy $\varepsilon=0.78 \mathrm{eV}$, as a function of the energy loss interval $\Delta E$, for 293 and $90 \mathrm{~K}$.

At $\Delta E=75 \mathrm{meV}$ in Fig. 6 , the relaxation times are the ones shown in Fig. 4 at $\varepsilon=0.78 \mathrm{eV}$. All of the points other than that at $\Delta E=75 \mathrm{meV}$ are our theoretical predictions for $\Delta E$ larger than the one used in our experiment (Fig. 6). For example, as one can see from Fig. 6 , the time of about 1 ps is needed for electrons initially at $\varepsilon=0.78 \mathrm{eV}$ to lose $0.4 \mathrm{eV}$ by the electron-phonon scattering at $90 \mathrm{~K}$, while, at $293 \mathrm{~K}, 1.25$ ps are needed.

One should note that, for sufficiently small $\Delta E$ values, $\tau_{E}$ depends almost linearly on $\Delta E$, and so the theoretical predictions shown in Fig. 4 can be simply rescaled to obtain the relaxation times for other experimental resolutions, provided that the latter do not exceed $0.1 \mathrm{eV}$ (see the appendix for details).

\section{Discussion: The roles of Fröhlich interaction and of intervalley scattering}

It is generally admitted that the initial relaxation of sufficiently hot electrons excited into the conduction band of a semiconductor is due to the interaction with zone-boundary (intervalley) phonons [29,43,44]. Although GaAs is a polar semiconductor, at sufficiently high excess energies the main electron-phonon scattering channel is due to interactions with intervalley phonons, as was first shown in Ref. [29] by semiempirical pseudopotential method and later confirmed by $a b$ initio calculations in Refs. $[8,13,16,17]$.

The energy relaxation of hot electrons in polar semiconductors is often attributed, in the literature, to the interaction with polar-optical phonons (Fröhlich interaction) [45-47], In the past, the role of the intervalley scattering in the energy relaxation of hot electrons was not clear. While, in Ref. [41], the intervalley scattering was suggested as an additional mechanism of the energy relaxation, in Ref. [42], intervalley scattering was viewed as the mechanism slowing down the energy relaxation of hot electrons. However, the results of Refs. $[8,13,16,17]$ provide a clear indication that intervalley scattering yields an efficient energy relaxation mechanism, which is expected to be the dominant one at sufficiently high excess energies. The analysis of our results presented below has confirmed the indication.

\section{Predominance of the energy loss at any temperature}

As already discussed in previous sections, the energy relaxation time $\tau_{E}$ depends on the difference between $R^{\text {loss }}$ and $R^{\text {gain }}$ [Eq. (6)].

In Fig. 7, we show separately the calculated energy loss and gain rates at two different temperatures, as a function of the excess energy. We note that energy loss is due to phonon emission, while energy gain is due to phonon absorption [Eqs. (1), (2), and (6)]. As can be seen in Fig. 7, the loss of energy by hot electrons due to phonon emission is always dominating over the energy gain, because the Bose-Einstein factor $n_{\mathbf{q}}^{\lambda}(T)+1$, which is associated with emission, is always larger than $n_{\mathbf{q}}^{\lambda}(T)$, the one for absorption. While at $293 \mathrm{~K}$, the energy gain rate behavior is similar to the loss one (although 


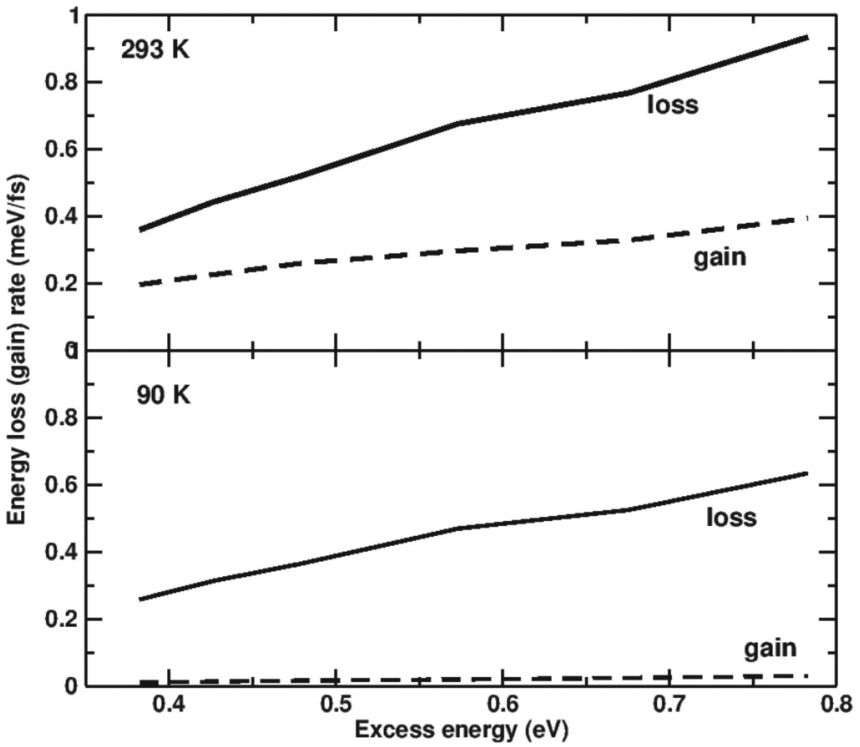

FIG. 7. Calculated total energy loss $R^{\text {loss }}$ (solid line) and gain $R^{\text {gain }}$ (dashed line) rates as a function of the excess energy. Top panel: 293 K. Bottom panel: 90 K. Calculations done for an initial electronic state in the $L$ valley.

the gain rate is much smaller), at $90 \mathrm{~K}$, absorption is suppressed due to the Bose-Einstein coefficient [Eq. (2)], and the energy gain rate becomes very small.

\section{Growing importance of intervalley scattering at high excess energy}

As the excess energy grows, the energy relaxation time becomes faster. The excess energy dependence of the energy relaxation time is mostly due to the growing density of final electronic states in the energy conservation law for the emitted phonons [Eqs. (1) and (9)]. In particular, scattering by intervalley phonons becomes more important as the excess energy grows. Indeed, in Fig. 8, we show the effective frequencies of emitted and absorbed phonons, calculated with Eqs. (3) and (4) as a function of excess energy, at two different temperatures.

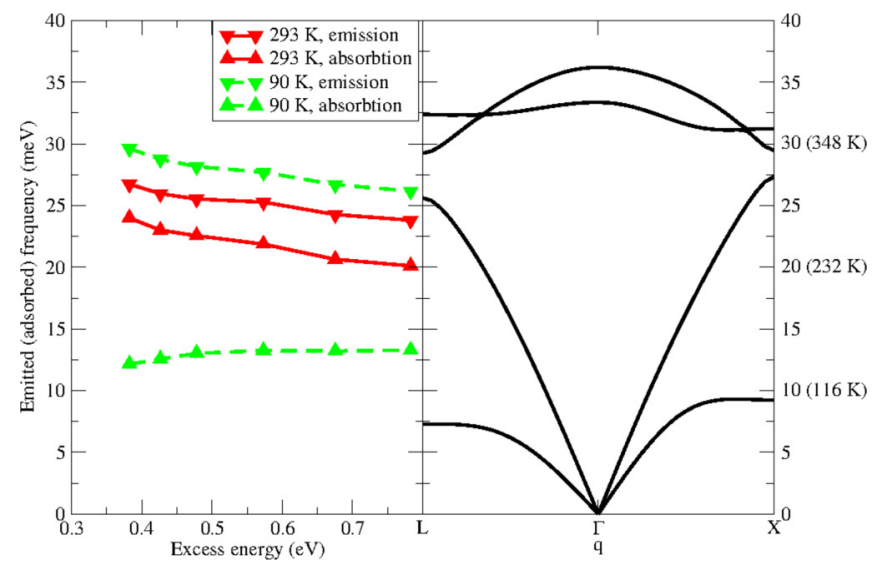

FIG. 8. Left panel: effective frequencies of emitted and absorbed phonons as a function of excess energy $(\mathrm{meV})$. Solid red lines: results at $293 \mathrm{~K}$. Green dashed lines: results at $90 \mathrm{~K}$. Triangles down: emission. Triangles up: absorption. Right panel: GaAs phonon dispersion.

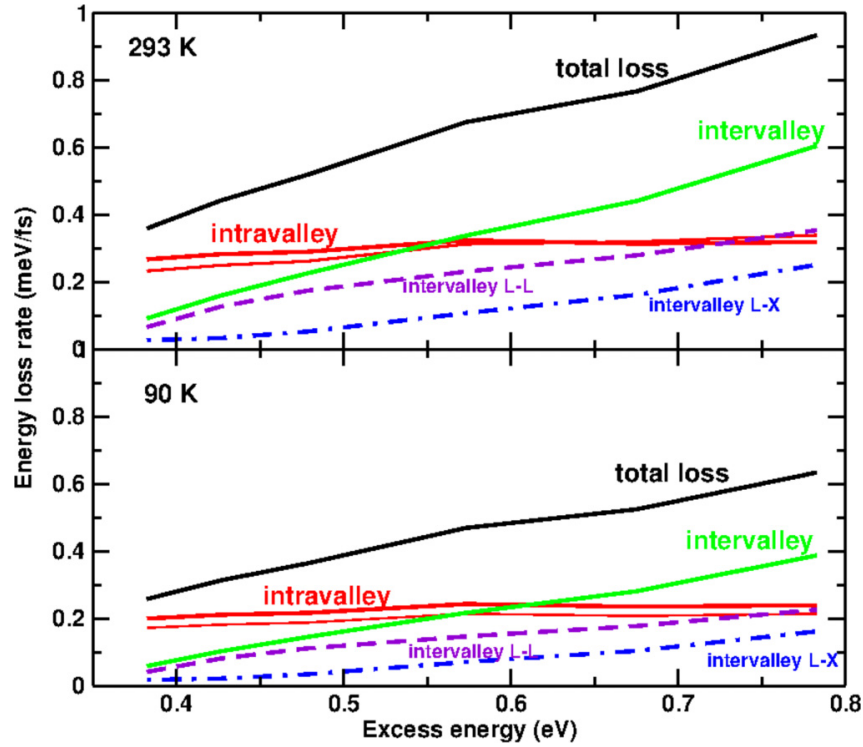

FIG. 9. Contributions to the energy loss rate $\left(R^{\text {loss }}\right)$ as a function of the excess energy. Top panel: $293 \mathrm{~K}$. Bottom panel: $90 \mathrm{~K}$. Calculations done for an initial electronic state in the $L$ valley. Solid black line: total energy loss rate. Thick red line: contribution to the energy loss (CEL) due to intravalley scattering within the $L$ valley, $|\mathbf{q}| \leqslant 0.252 \pi / a$. Thin red lines: CEL due to the Fröhlich interaction. Green lines: CEL due to intervalley scattering. Dashed violet lines: CEL due to intervalley scattering from $L$ to other $L$ valleys ( $L-L$ scattering contribution). Dot-dashed blue lines: CEL due to intervalley scattering from $L$ to $X$ valleys ( $L-X$ scattering contribution).

As one can see, the effective frequencies of both emitted and absorbed phonons at $293 \mathrm{~K}$ are close to the ones of zone-border acoustic phonons. They are found to be significantly lower than the frequency of polar optical phonon at $\Gamma(36 \mathrm{meV})$.

The effective frequencies of both emitted and absorbed phonons decrease with increasing excess energies, reflecting the increase of the relative importance of intervalley scattering. At $90 \mathrm{~K}$, the frequencies of absorbed phonons are very small, due to the absence of phonon population but for very lowenergy phonons. The emitted phonons at $90 \mathrm{~K}$ are found to be higher in energy than the ones at $293 \mathrm{~K}$. At low excess energies, one can see that the effective frequency of the emitted phonons at $90 \mathrm{~K}$ becomes higher than the ones of the most energetic intervalley phonons, reflecting the important role of the Fröhlich coupling in this case, as we show below. It should be noted that the effective frequency of the emitted phonons never reaches the value of the longitudinal optical phonon frequency at $\Gamma(36 \mathrm{meV})$, as scattering channels other then Fröhlich interaction are always present.

\section{Analysis of the scattering channels}

In order to further analyze the roles of different scattering channels in the energy loss, in Fig. 9, we show the energy loss rates at 293 and $90 \mathrm{~K}$, calculated taking into account all electron-phonon scattering events (solid black lines), as well as the ones in which only Fröhlich coupling is taken into account (thin red lines). While in the former case, the energy loss rate changes strongly with the excess energy, in the latter one the energy loss rate due to Fröhlich coupling stays quasiconstant. Moreover, one can see that the energy loss 
attributed to intravalley scattering (in this case, we have limited our calculation to phonons with $|\mathbf{q}| \leqslant 0.252 \pi / a$ ) (thick red lines) is almost entirely due to Fröhlich coupling, as the thick and thin red lines almost coincide in both panels of Fig. 9.

As to the intervalley scattering, we show the total intervalley contribution (green lines) as well as separate contributions from the intervalley scattering to the $L$ valley (violet dashed lines) and to the $X$ valley (dot-dashed blue lines) [48]. The contribution of intervalley scattering to the $\Gamma$ valley has been found very small and is not shown. As one can see, the intervalley scattering contributions to the energy loss rate grow rapidly with the excess energy.

\section{Role of the Fröhlich coupling}

Let us now turn to the relative importance of the Fröhlich coupling in the emission rate (not shown) and in the energy loss rate (Fig. 9). As expected, the relative role of Fröhlich coupling (thin red lines) varies with the excess energy. While at low excess energies, it is clearly the dominant energy loss mechanism, whereas at high excess energy the intervalley scattering (green lines) becomes the dominant mechanism, due to the large density of final electronic states available for this scattering process, notwithstanding the fact that the emitted intervalley phonon energies (typically around $20 \mathrm{meV}$ ) are significantly smaller than the energy of polar optical phonon (36 meV). As shown in Fig. 9, the main intervalley scattering mechanisms are scattering between equivalent $L$ valleys ( $L-L$ scattering, dashed violet lines) and scatterings between $L$ and $X$ valleys ( $L-X$ scattering, dash-dotted blue lines).

The relative role of the Fröhlich interaction in the energy loss is slightly more important at $90 \mathrm{~K}$ than at $293 \mathrm{~K}$, at all excess energies, because the population of low-energy acoustic phonons is the most sensitive one to this increase of temperature.

Finally, the relative contribution of the Fröhlich interaction to the energy loss rate of Fig. 9 is found to vary from $65 \%$ to $35 \%$ in the studied range of excess energies, at $293 \mathrm{~K}$. At $90 \mathrm{~K}$, this contribution varies from $67 \%$ to $34 \%$. Thus, we find that, as expected [42,45-47], the Fröhlich interaction provides the dominant mechanism of the energy relaxation at low excess energies. For hot electrons, on the contrary, we find that the dominant contribution comes from the energy loss by intervalley scattering. Indeed, at high excess energies, the total contribution of the intervalley scattering represents as much as $65 \%$ of the energy loss rate at $293 \mathrm{~K}$ and $62 \%$ at $90 \mathrm{~K}$.

\section{Concluding remarks}

Thus, one can conclude that, as could be expected from the results of previous recent calculations $[8,13,16,17]$, the Fröhlich interaction plays a determinant role in energy relaxation only at low excess energies. At high excess energies, on the contrary, the energy relaxation is governed mostly by the interaction with intervalley phonons.

\section{CONCLUSION}

In conclusion, we have presented the results of a combined theoretical and experimental work, in which we have studied the energy relaxation of hot electrons in GaAs, in a wide range of excitation energies and at two different temperatures. The energy relaxation times are shown to be due to electronphonon interaction, and the agreement between theory and experiment is remarkably good. Interestingly, the relaxation times are found to be faster at lower temperatures, in wide contrast with the temperature behavior of the momentum relaxation rate. This temperature dependence is explained by the difference in the density of final electronic states available for emission and absorption, and is shown to come from the most energetic phonons with energies around $30 \mathrm{meV}$. Finally, it is demonstrated that the interaction with polar optical phonon (Fröhlich coupling) is responsible for the energy relaxation of hot electrons only at low excess energies. At high excess energies, on the contrary, the intervalley scattering is shown to be the main mechanism for the energy loss.

\section{ACKNOWLEDGMENTS}

This work was supported by LABEX PALM (ANR-10LABX-0039-PALM, Project Femtonic) and by the Graphene Flagship. Results have been obtained with the QUANTUM ESPRESSO [39,49] and WANNIER90 [50] packages. We acknowledge support from the French DGA and from the Chaire Energie of the Ecole Polytechnique, and computer time has been granted by GENCI (Project 2210) and by Ecole Polytechnique through the LLR-LSI project. This work was also supported by the Japan Society for the Promotion of Science (JSPS) KAKENHI Grant No. 24000006.

\section{APPENDIX: LINEAR DEPENDENCE OF THE ENERGY RELAXATION TIME ON THE ENERGY LOSS INTERVAL}

As we mentioned in the main text, the energy relaxation time $\tau_{E}$ can be considered as depending almost linearly on the energy loss interval $\Delta E$ (or, equivalently, on the experimental resolution) for sufficiently small $\Delta E$ values. Indeed, in Fig. 10,

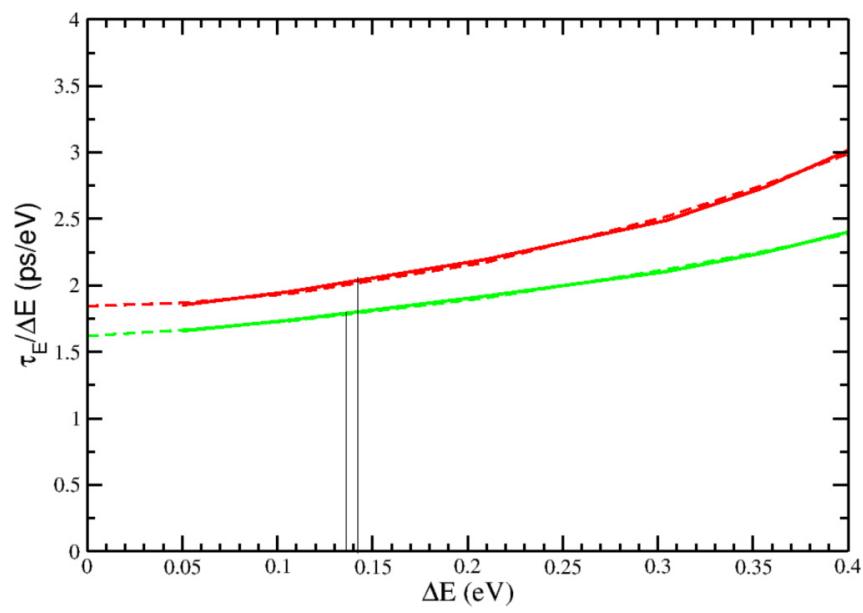

FIG. 10. Calculated energy relaxation time of hot electrons in $\mathrm{GaAs}$, for $\varepsilon=0.78 \mathrm{eV}$ with respect to $\mathrm{CBM}$, as a function of the energy loss interval $\Delta E$, divided by $\Delta E$ [Eq. (A1)]. Solid red curve: 293 K. Solid green curve: 90 K. Dashed thin curves: quadratic fit of the calculated data. Calculations done for an initial electronic state in the $L$ valley. 
we plot a quantity $\tau_{E} / \Delta E$ :

$$
\tau_{E} / \Delta E=\frac{1}{\Delta E} \int_{\varepsilon-\Delta E}^{\varepsilon} d \varepsilon^{\prime} \frac{1}{\langle\omega\rangle\left(\varepsilon^{\prime}\right) \Gamma\left(\varepsilon^{\prime}\right)} .
$$

In Fig. 10, our calculation results are presented by solid curves, while the dashed curves are obtained by a quadratic fit of the former, in order to estimate a value for $\tau_{E} / \Delta E$ at zero $\Delta E$. As one can see in Fig. 10, although the curves are not horizontal, they can be considered as such for sufficiently small $\Delta E$. For instance, for $\Delta E<0.1 \mathrm{eV}$, the deviation from zero $\Delta E$ value is only $3 \%$. Vertical lines in Fig. 10 indicate the $\Delta E$ values for which the $\tau_{E} / \Delta E$ deviates by $10 \%$ from zero $\Delta E$ value.
[1] C. Delerue and M. Lannoo, Nanostructures: Theory and Modeling, Nanoscience and Technology (Springer Verlag, Berlin, 2004).

[2] A. Polman and H. A. Atwater, Nat. Mater. 11, 174 (2012).

[3] F. Rossi and T. Kuhn, Rev. Mod. Phys. 74, 895 (2002).

[4] J. Shah, Hot Carriers in Semiconductor Nanostructures: Physics and Applications (Academic Press, Boston, 1992).

[5] J. Kanasaki, H. Tanimura, and K. Tanimura, Phys. Rev. Lett. 113, 237401 (2014)

[6] E. Papalazarou, J. Faure, J. Mauchain, M. Marsi, A. TalebIbrahimi, I. Reshetnyak, A. van Roekeghem, I. Timrov, N. Vast, B. Arnaud, and L. Perfetti, Phys. Rev. Lett. 108, 256808 (2012).

[7] H. Tanimura, J. Kanasaki, K. Tanimura, J. Sjakste, N. Vast, M. Calandra, and F. Mauri, Phys. Rev. B 93, 161203(R) (2016).

[8] J. Sjakste, N. Vast, M. Calandra, and F. Mauri, Phys. Rev. B 92, 054307 (2015).

[9] C. Verdi and F. Giustino, Phys. Rev. Lett. 115, 176401 (2015).

[10] J. Sjakste, I. Timrov, P. Gava, N. Mingo, and N. Vast, in Annual Review of Heat Transfer (Begell House, Danbury, CT, 2014), Vol. 17, p. 333.

[11] M. Calandra, G. Profeta, and F. Mauri, Phys. Rev. B 82, 165111 (2010).

[12] A. Marini, J. Phys. Conf. Ser. 427, 012003 (2013).

[13] J. J. Zhou and M. Bernardi, Phys. Rev. B 94, 201201 (2016).

[14] N. Vast, J. Sjakste, G. Kané, and V. Trinité, Electrons, Phonons, and Their Coupling within the Density Functional Theory, Simulation of Transport in Nanodevices Vol. 1 (ISTE, London, United Kingdom, 2016).

[15] F. Giustino, Rev. Mod. Phys. 89, 015003 (2017), and references therein.

[16] T.-H. Liu, J. Zhou, B. Liao, D. J. Singh, and G. Chen, Phys. Rev. B 95, 075206 (2017).

[17] M. Bernardi, D. Vigil-Fowler, C. S. Ong, J. B. Neaton, and S. G. Louie, Proc. Natl. Acad. Sci. U.S.A. 112, 5291 (2015).

[18] J. Sjakste, V. Tyuterev, and N. Vast, Appl. Phys. A 86, 301 (2007).

[19] J. Sjakste, N. Vast, and V. Tyuterev, Phys. Rev. Lett. 99, 236405 (2007).

[20] F. Giustino, S. G. Louie, and M. L. Cohen, Phys. Rev. Lett. 105, 265501 (2010).

[21] M. Bernardi, D. Vigil-Fowler, J. Lischner, J. B. Neaton, and S. G. Louie, Phys. Rev. Lett. 112, 257402 (2014).

[22] S. Poncé, Y. Gillet, J. L. Janssen, A. Marini, M. Verstraete, and X. Gonze, J. Chem. Phys. 143, 102813 (2015).

[23] J. P. Nery and P. B. Allen, Phys. Rev. B 94, 115135 (2016).

[24] S. Zollner, J. Kircher, M. Cardona, and S. Gopalan, Solid-State Electron. 32, 1585 (1989).
[25] A. R. Goñi, A. Cantarero, K. Syassen, and M.Cardona, Phys. Rev. B 41, 10111 (1990).

[26] D. Karaiskaj, J. A. H. Stotz, T. Meyer, M. L. W. Thewalt, and M. Cardona, Phys. Rev. Lett. 90, 186402 (2003).

[27] G. H. Li, A. R. Goñi, K. Syassen, and M. Cardona, Phys. Rev. B 49, 8017 (1994).

[28] D. Lüerßen, R. Bleher, and H. Kalt, Phys. Rev. B 61, 15812 (2000).

[29] S. Gopalan, P. Lautenschlager, and M. Cardona, Phys. Rev. B 35, 5577 (1987).

[30] C. Jacoboni and L. Reggiani, Rev. Mod. Phys. 55, 645 (1983).

[31] P. K. Basu, Phys. Lett. A 68, 459 (1978).

[32] B. Ridley, Quantum Processes in Semiconductors (Clarendon Press, Oxford, UK, 1993).

[33] D. Sangalli and A. Marini, Europhys. Lett. 110, 47004 (2015).

[34] D. Sangalli, S. Dal Conte, C. Manzoni, G. Cerullo, and A. Marini, Phys. Rev. B 93, 195205 (2016).

[35] Molina-Sánchez, D. Sangalli, L. Wirtz, and A. Marini, Nano Lett. 17, 4549 (2017).

[36] V. P. Zhukov, P. M. Echenique, and E. V. Chulkov, Phys. Rev. B 82, 094302 (2010).

[37] S. Sadasivam, M. K. Y. Chan, and P. Darancet, Phys. Rev. Lett. 119, 136602 (2017).

[38] V. Jhalani, J. Zhou, and M. Bernardi, Nano Lett. 17, 5012 (2017).

[39] S. Baroni, S. de Gironcoli, A. D. Corso, and P. Giannozzi, Rev. Mod. Phys. 73, 515 (2001).

[40] P. Vogl, Phys. Rev. B 13, 694 (1976).

[41] D.-S. Kim and P. Y. Yu, Phys. Rev. B 43, 4158 (1991).

[42] R. Clady, M. J. Y. Tayebjee, P. A. Aliberti, D. König, N. J. Ekins-Daukes, G. J. Conibeer, T. W. Schmidt, and M. A. Green, Progr. Photovoltaics: Res. Appl. 20, 82 (2012).

[43] C. J. Stanton and D. W. Bailey, Phys. Rev. B 45, 8369 (1992).

[44] J. Shah, B. Deveaud, T. C. Damen, W. T. Tsang, A. C. Gossard, and P. Lugli, Phys. Rev. Lett. 59, 2222 (1987).

[45] D. Bailey, C. Stanton, M. Artaki, K. Hess, F. Wise, and C. Tang, Solid-State Electron. 31, 467 (1988).

[46] Y. Feng, S. Lin, M. Green, and G. Conibeer, J. Appl. Phys. 113, 024317 (2013).

[47] J. Z. Zhang, J. Appl. Phys. 115, 203704 (2014).

[48] The scattering from $L$ to $X$ is not strictly zero for excess energies smaller than the bottom of the $X$ valley due to the use of Gaussian functions instead of $\delta$ ones in integrals (see main text).

[49] P. Giannozzi, O. Andreussi, T. Brumme, O. Bunau, M. B. Nardelli, M. Calandra, R. Car, C. Cavazzoni, D. Ceresoli, M. Cococcioni et al., J. Phys.: Condens. Matter 29, 465901 (2017).

[50] N. Marzari, A. A. Mostofi, J. R. Yates, I. Souza, and D. Vanderbilt, Rev. Mod. Phys. 84, 1419 (2012). 